\title{
Software Scripts for Quality Checking of High-Throughput Nucleic Acid Sequencers
}

BioTechniques 30:1300-1305 (June 2001)

\author{
G.R. Lazo, J. Tong, R. Miller, \\ C. Hsia, C. Rausch, Y. Kang, \\ and O.D. Anderson \\ USDA ARS Western Regional \\ Research Center, Albany, CA, \\ USA
}

\section{INTRODUCTION}

Capillary electrophoresis technology, featuring high-sensitivity detection through laser-induced fluorescence, has resulted in a number of easy-to-use, fully automated DNA sequencing systems for high-resolution separation of DNA fragments that eliminates many tedious tasks associated with DNA analysis. These systems are computer program controlled and are configurable for routine laboratory practices.

High-throughput sequencers run several samples simultaneously and automatically after sample preparation. The ABI PRISM ${ }^{\mathrm{TM}} 3700$ (Applied Biosystems, Foster City, CA, USA) can run the equivalent of eight 96 -well microtiter dishes per day, running 96 sam ples at a time. The CEQ 2000 (Beckman Coulter, Fullerton, CA, USA), an eight-channel capillary machine, can run one 96-well microtiter dish in approximately $24 \mathrm{~h}$. Other automated sequencers are available, with most handling the samples in microtiter format dishes. Some sequencing instruments also handle 384-well microtiter dishes.

In high-throughput sequencing, most samples in any given run are handled collectively for a number of different analyses, such as expressed sequence tag (EST) production, genome sequencing and mapping, clone contig building, and other methods. There are several packages constructed for working with a large collection of sequences, which will be passed on for further analyses and processing. One program that has gained considerable acceptance in the first step of sequence processing is the phred molecular biology program $(3,4)$. The phred program reads sequencing trace file data and creates files for the sequencing base calls and for the quality measurement of each base call. The files once created, usually in FASTA format, are used by a series of other molecular biology programs. The program options are varied and may be adjusted for different laboratory applications.

The high volume of data generated by these automated sequencers requires the development of software tools to aid the collection of sequence data and to assess the quality of the data before passing it on for further processing. Discussed here is a small collection of Perl scripts, designated sequence quality plate reporter $(S Q P R)$ scripts, which when placed under an inter/intranet Web server allow the scientist to spot check the quality of the sequence data being generated by automated sequencers. These scripts also generate summary files to archive the progress of a research group's sequencing efforts. In our case, the sequencers were being used to screen EST data for a wheat (Triticum aestivumL.) genomics project, and the programs allowed the visualization of the quality of sequencing runs. It is possible to customize the scripts to match the particular needs of handling the data in diverse laboratory environments.

\section{MATERIALS AND METHODS}

Data were generated from two dif- 
ferent automated sequencers that handle samples prepared in microtiter dishes arrayed in a 96-well format, the ABI PRISM 3700 and the CEQ 2000. The ABI PRISM 3700 is also able to accom modate 384-well dishes. The phred program $(3,4)$ used initially to analyze the data from the trace files was able to read both the ABI (.ab1) and SCF (.scf) trace file formats. The phred program can also read files in MegaBACE ${ }^{\circledR}$ (Amersham Pharmacia Biotech, Piscataway, NJ, USA) ABD/ESD chromatogram files, automatically detecting the file format and whether the chromatogram file was compressed by "gzip" or UNIX ${ }^{\circledR}$ "compress" com mands. The phred program is able to recognize chromatograms generated under several primer/chemistry conditions, but the appropriate entries must be added to the PHRED_PARAMETER_FILE and configured as specified by the authors of phred.

After sequence data were generated by the automated sequencer, typically on a Microsoft ${ }^{\circledR}$ or Macintosh ${ }^{\circledR}$ platform machine, the directory folder was transferred to a UNIX platform machine by a file transfer protocol (FTP) program. The directory folder was placed on the UNIX machine in a location specified for the $S Q P R$ programs, termed the trace file data directory $(T F D D)$, and the scripts were used to process and display data (Figure 1). To initiate processing, a Web server was used to execute one of the cgi-bin scripts from a preset Web address (e.g., http://hostname/cgibin/dirdisplay.cgi) to view the data folders available for processing. Netscape ${ }^{\circledR}$ Communicator $^{\circledR}$ (version 4.7) or $\mathrm{Mi}$ crosoft Internet Explorer ${ }^{\circledR}$ (version 5.0) Web browsers were used to view the directory folders available, initiate analyses, and to view the results that followed. Upon selecting a directory folder for analysis, the phred program would read and process trace files in the selected directory folder; then, $S Q P R$ scripts would further be used to read results of the phred output and display the calculations onto a hypertext mark-up language (HTML specification version 4) page. Results were archived into a folder, designated the phred-output data directory $(P O D D)$, so that the phred program would only need to be run once per sam ple. A separate script was used to display the analyzed archives, allowing selection of the $P O D D$ data via the Web browser. Results were displayed with some calculated measurements (Figure 2) for the run and with color-coded HTML graphical displays of the microtiter dish (Figure 3).

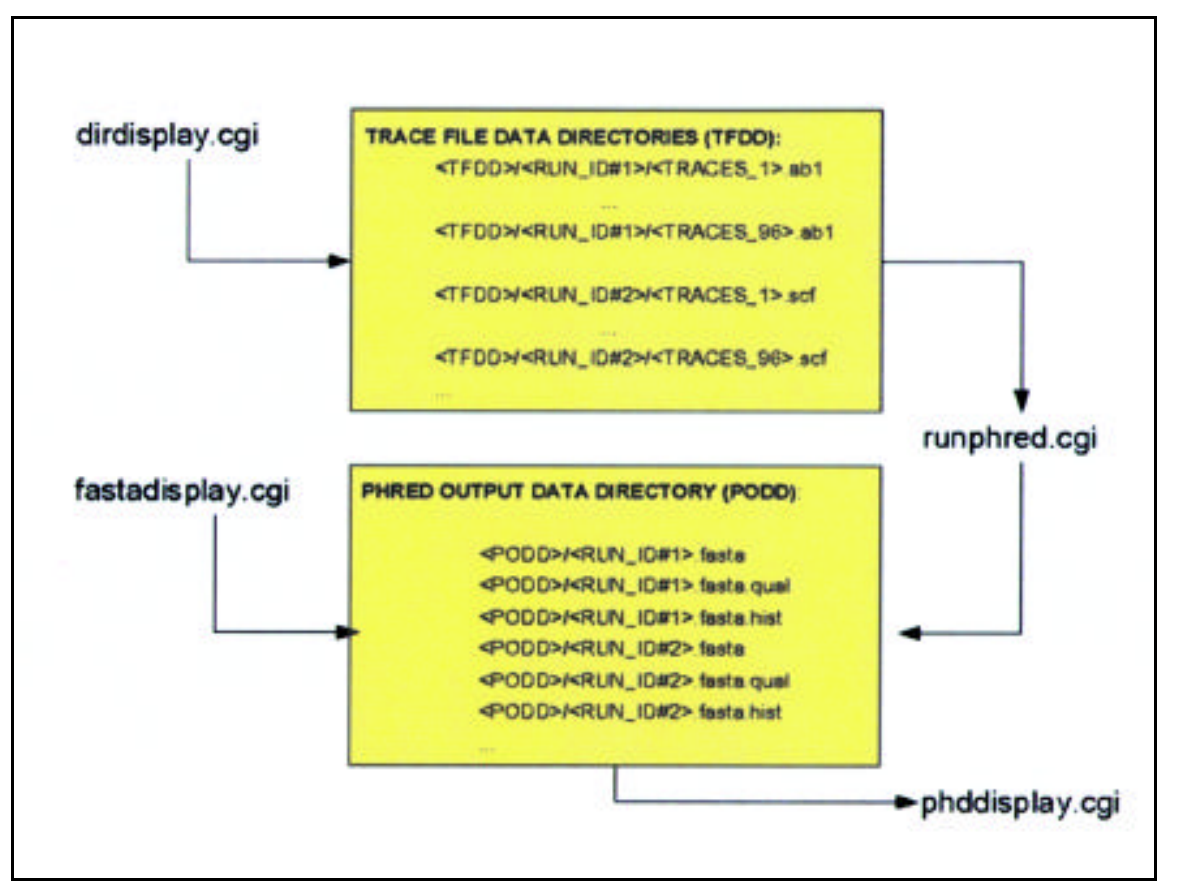

Figure 1. Flow diagram of how $S Q P R$ cgi-bin scripts interact.
For the scripts to work correctly, a sample naming syntax was developed and followed. The naming system primarily keyed upon the plate identity, well coordinates of 96 independent samples from a 96-well (or 384-well) formatted microtiter dish, an indicator designating if the sample was a control or test sample, an indicator designating the oligonucleotide primer used for the sequencing reaction, other comments or indicators for laboratory tracking, and the capillary number used by the automated sequencer (if available). Some of the naming fields, such as the capillary position, are in fixed positions as generated by the automated sequencer and are set as configured by the manufacturer. Further specifics on the naming syntax are explained in the software package, which is available for download from the Internet.

The UNIX operating system used in this case ran under the Solaris ${ }^{\circledR} 7$ (Sun Microsystems, Palo Alto, CA, USA) operating system, and utilized the Apache $^{\circledR}$ HTTP Server 1.3.6 (The Apache Software Foundation, Forest Hill, MD, USA), and phred 0.990722.h $(3,4)$. Perl scripts were executed under the Solaris environment with Perl 5.005_03 (5).

\section{RESULTS}

Four Perl script programs were created for handling trace files generated by the automated sequencers. All of the programs ran as executables under a Web server and displayed the results as HTML-encoded pages. One script (dirdisplay.cgi) listed unprocessed data folders, termed $T F D D$, containing ABI or SCF trace files. Another script (runphred.cgi) executed the phred program with set parameters to read the trace files and place the output data into a designated folder, termed $P O D D$. With data in the $P O D D$, another script (fastadisplay.cgi) listed the data files available for viewing; selection of the data file using the browser interface activated the data-viewing script (phddisplay.cgi). The data-viewing script read data from the result files in the $P O D D$ and displayed them in a graphical fashion as color-coded tables (Figures 2 and 3 ).

Statistical measures for the entire 
run included: $(i)$ a count of samples that met or exceeded a quality read length requirement, (ii) the average starting base position of the quality read length for control and test samples, (iii) the average quality read length for control and test samples, (iv) the number of failed samples, ( $v)$ the average phred score, (vi) the percent of phred calls in a quality read length that fell below a score of 20, (vii) the total number of bases counted for all samples in a given run, and (viii) a histogram showing the distribution of quality read lengths. Also included were displays showing for each plate, statistics for each individual sample well to include item (i) quality read length, (ii) average phred score, (iii) percent calls below a phred score of 20 , and (iv) capillary number of the automated sequencer used (where applicable, such as with the ABI PRISM 3700). The quality read length display (Item $i$ ) is shown in Figure 3; the other items not shown (Items $i i-i v$ ) have a similar appearance except for the different sample statistics displayed. Some sequencers, such as the CEQ 2000, have fixed capillary positions and do not display this statistic.

An added option in the phddisplay. cgi display is a text box window for annotation (Figure 2) so that comments can be added before printing if desired (these comments are not saved). A button was also set to append and store the run statistics to another file (passfile.txt or failfile.txt) that was used to store summaries of sequencing runs. Supplemental Perl scripts were also included to make adjustments for different orders of sample loading from 384-well plates (trace.cgi). Selecting a well position from the HTML table produced by the phddisplay.cgi script activated a Java $^{\circledR}$ (Sun Microsystems) applet that displayed the raw trace file in a pop-up browser window (1) (Figure 4). If sequence files did not conform to the sample naming syntax, an error message was displayed. If empty wells appeared in the display, an option button was displayed allowing the viewer to re-run the phred analysis (redo.cgi). A file was also included to point to variables specific to the computing environment (GlobalVariables.lib) and direct file input to the HTML pages (subparseform.lib) (2).

\section{DISCUSSION}

We have developed a software suite for assessing sequencing performance and quality. The $S Q P R$ scripts utilize the phred program to initiate trace file processing and use the output files to generate HTML pages containing statistical and quality measurements. The data files as stored may be used for further processing by other molecular biology packages.

The automated sequencer systems used in developing the $S Q P R$ scripts both operated using computers running Microsoft Windows ${ }^{\mathrm{TM}}$ NT (version 4.0) operating systems, and most of the post-sequencing processing was done under a UNIX operating system. It may be possible to streamline these procedures further by using cross-platform disk partitioning or disk file sharing (using software packages such as SAMBA; http://www.samba.org), and integrating automated processing using the UNIX "crontab" feature. Versions of the Apache HTTP Server and Perl are able to operate under a Windows operating system. The phred software is typically executed under UNIX; however, there are modified phred versions (CodonCode, Dedham, MA,

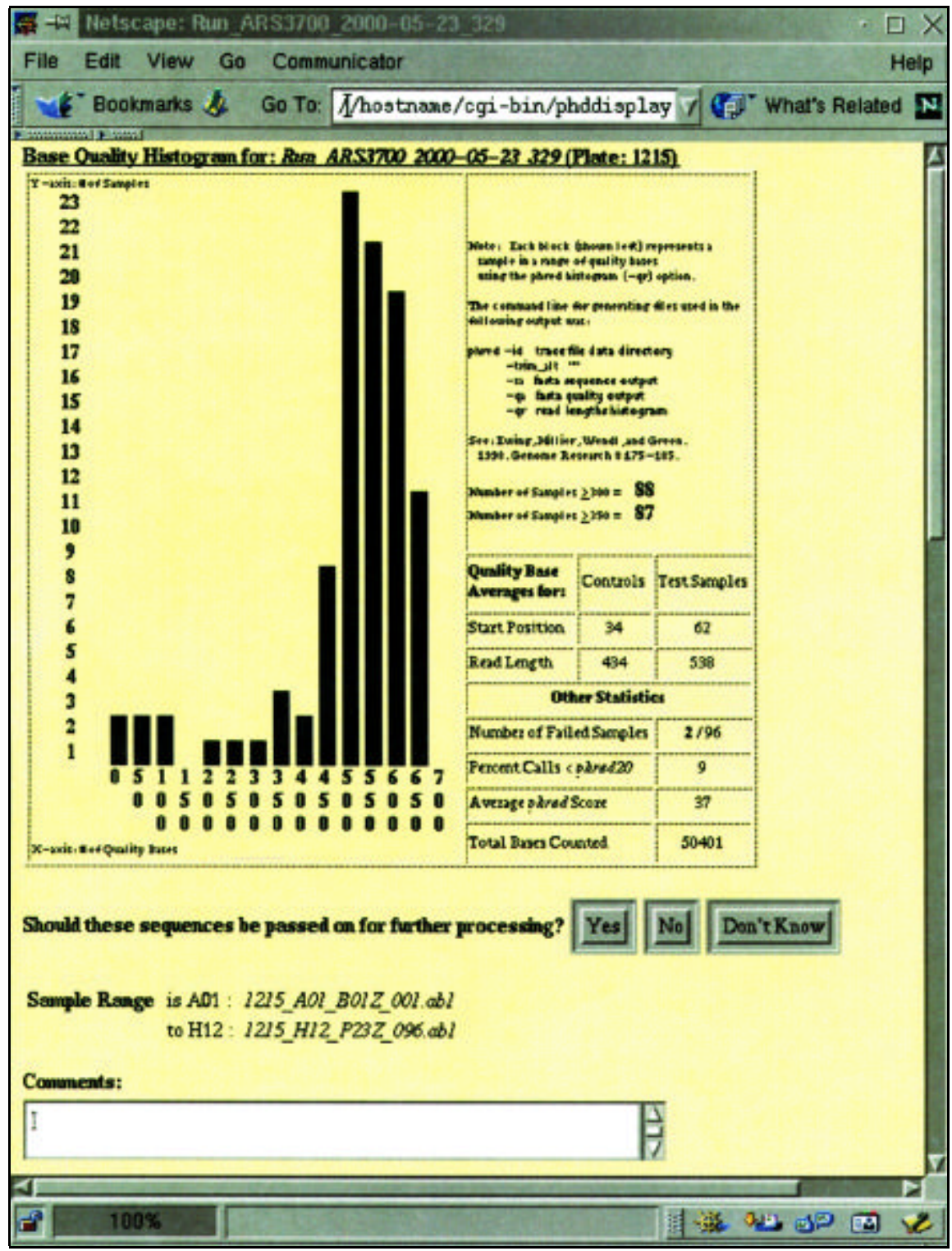

Figure 2. Histogram and statistics display of results. 


\section{BioComputing}

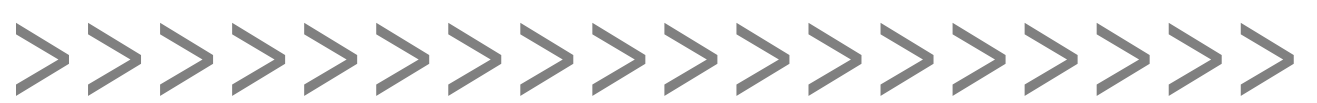

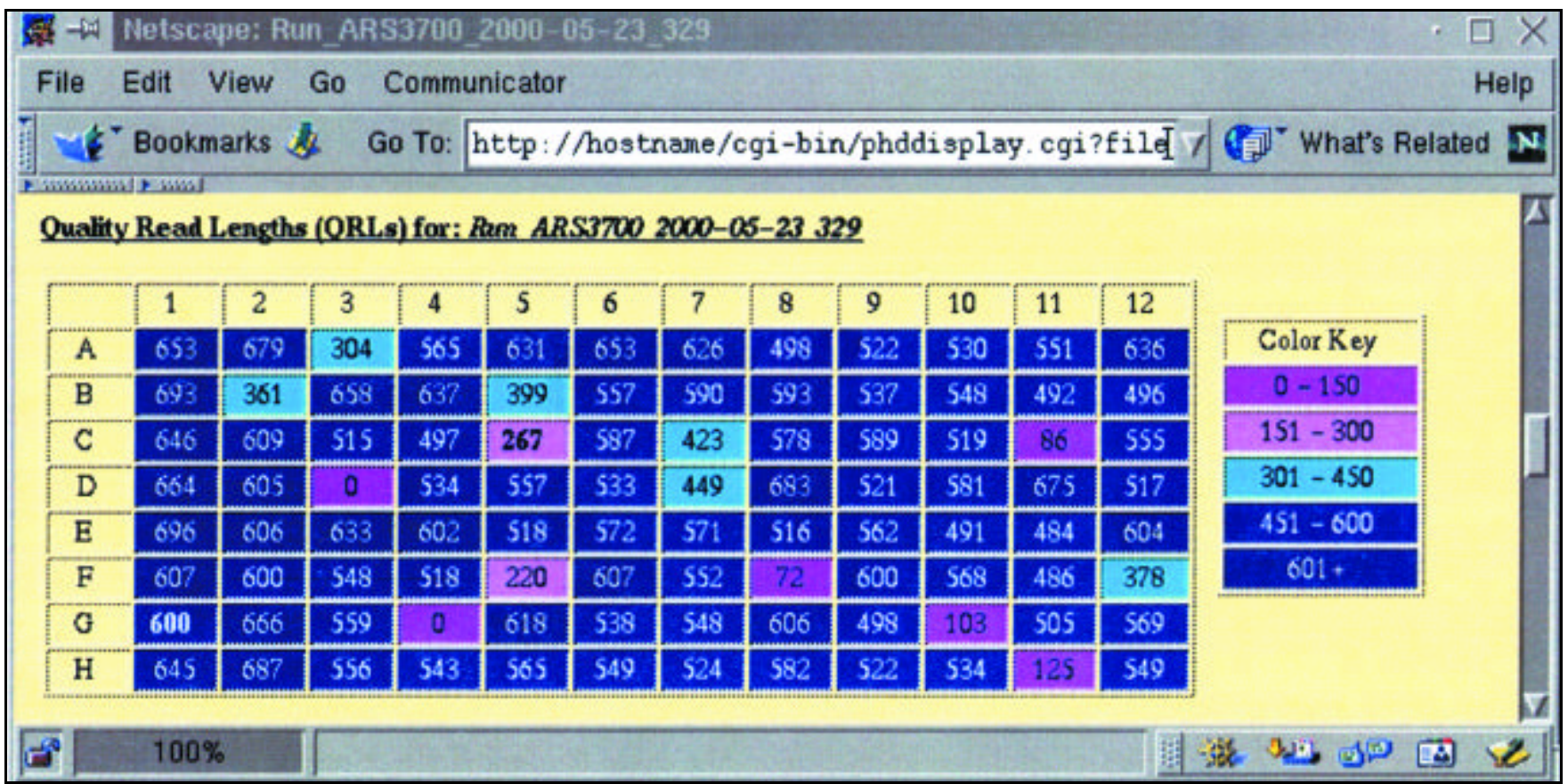

Figure 3. Graphical display of well quality measurements. Similar displays are also available for average phred score, percent base calls below a phred score of 20, and capillary number used by the automated sequencer. 


\section{$\gg>>>>>>>>>>>>>>>>>>>>>>$}

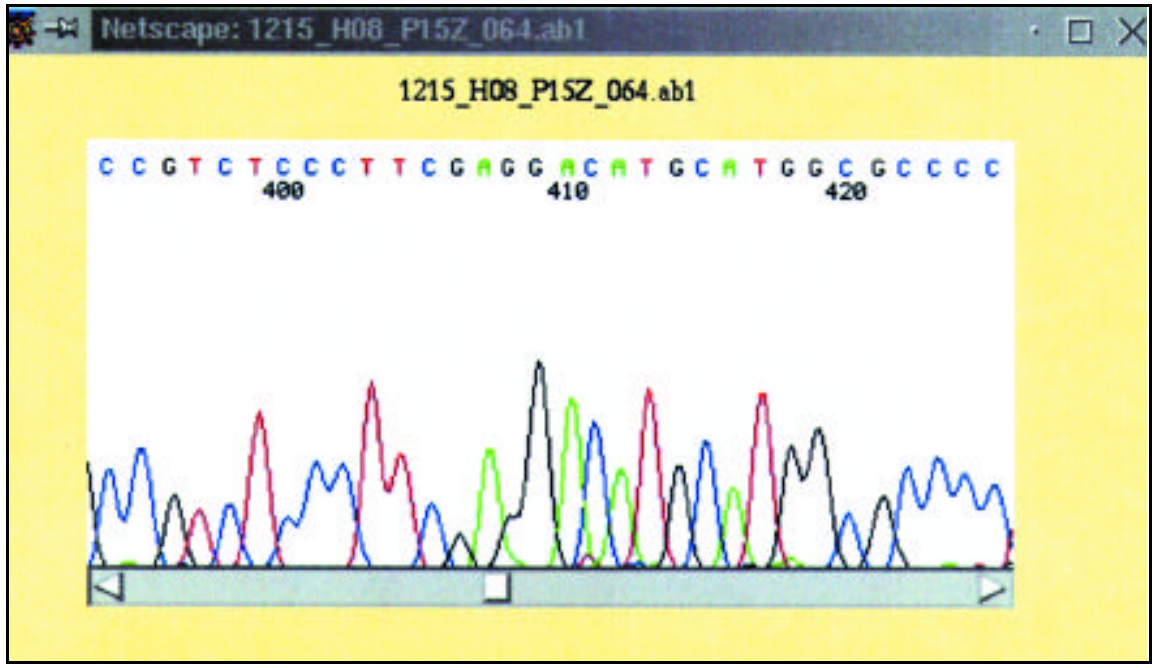

Figure 4. Trace file applet display (1).

USA) that operate under the Windows environment. The source code for phred, available by agreement from the authors, may be used to create the executable program using $\mathrm{C}^{++}$compilers for Windows.

Uncompressed trace files for each sample are approximately 220000 bytes in size, so computer hard disk storage may be a limiting factor on some systems. Our laboratory archives trace files onto CD-R disks but maintains the phred output files used by the $S Q P R$ scripts locally to allow checking past sequencing runs with the graphical interface (these are found in the $P O D D$ ).

Other automated sequencers, such as the ABI PRISM 310 single-channel electrophoresis system or other com puter-controlled sequencers (e.g., ABI PRISM373/377), may utilize the $S Q P R$ scripts, provided that a similar naming syntax is used. The ABI PRISM 310 is usually configured in a 48 -well $(6 \times 8)$ format; however, samples retaining coordinates from a 96-well dish should display correctly with the default $S Q P R$ settings. Adjustments were also made for reading 384-well microtiter dishes to accommodate the different sample loading procedures used for the ABI PRISM 3700 sequencer. In this case, samples were read either in quadrants of the dish (A1-P6, A7-P12, A13-P18, and A19-P24), or by offset position (A1-O23, A2-O24, B1-P23, and B2$\mathrm{P} 24)$. Samples loaded by quadrant were displayed using a $6 \times 16$ display instead of the default $8 \times 12$ display, and rows and columns were labeled accordingly.

The version of the Java applet used to view the trace files (1) gives a general idea of the quality of the trace file. There are other trace file-viewing applets written that may be better tuned for displaying a wide variety of traces generated from different sequencing systems. This may be needed because some of the trace values differ from those called by the phred program, which has been trained on several different automated systems (ABI PRISM 373/377/3700 and MegaBACE). At the time of this writing, the phred program had not been trained on the CEQ 2000 system by the authors of phred, but adjusting and testing the chemistry settings of the PHRED_PARAMETER_ FILE allowed finding a setting that closely matched calls made from the CEQ 2000 system.

These scripts have been useful in assessing sequencing performance and quality. On occasion, these scripts have been of value in troubleshooting problems that occur in a high-throughput operation, usually identifying troublesome library preparations. The convenience of having this data accessible over the Web has allowed, when needed, sharing and discussing sequencing information with other remotely located scientists. If needed, access to the sequencing run information may be protected using security methods in- cluded with the Apache HTTP Server. Sample displays and Perl scripts may be viewed and downloaded from the authors' Web site (http://wheat.pw. usda.gov/demos/SQPR/). A bulletin board has been set up at this site for feedback on these scripts.

\section{ACKNOWLEDGMENTS}

Results shown here are part of the United States Department of Agriculture, Agricultural Research Service Current Research Information System (CRIS no. 0149126), and partial funding was provided by the National Science Foundation Award (NSF no. 9975989).

\section{REFERENCES}

1.Buehler, E. 1996. Chromatogram Applet, Release 1, 6/30/96. (Software, Unpublished.)

2.Castro, E. 1999. Perl and CGI for the World Wide Web: Visual QuickStart Guide. Peachpit Press, Berkeley, CA.

3.Ewing, B. and P. Green. 1998. Base-calling of automated sequencer traces using phred. II. Error probabilities. Genome Research 8:186194.

4.Ewing, B., L. Hillier, M.C. Wendl, and P. Green. 1998. Base-calling of automated sequencer traces using phred. I. Accuracy assessment. Genome Res. 8:175-185.

5.Wall, L. and R.L. Schwartz. 1991. Program ming Perl. O'Reilly \& Associates, Inc., Sebastopol, CA.

Received 15 August 2000; accepted 23 April 2001.

\author{
Address correspondence to: \\ Dr. Gerard R. Lazo \\ USDA ARS Western Regional Research Center \\ 800 Buchanan Street \\ Albany, CA 94710-1105, USA \\ e-mail: lazo@pw.usda.gov
}

